\title{
Exercise-induced airway narrowing in army recruits with a history of childhood asthma
}

\author{
D.G. Sinclair*, M.M. Sims**, N.A. Hoad*, C.R. Winfield*
}

Exercise-induced airway narrowing in army recruits with a history of childhood asthma. D.G. Sinclair, M.M. Sims, N.A. Hoad, C.R. Winfield. CERS Journals 1995.

ABSTRACT: The aim of this study was to determine whether the addition of cold, dry, inspired air to maximum heart rate (MHR) exercise increased the number of subjects demonstrating abnormal airway narrowing, when applied to symptom-free army recruits with a history of asthma in childhood.

One hundred recruits with a history of asthma in childhood underwent two exercise challenges to maximum heart rate, breathing either room air or cold dry air in randomized order. The percentage fall index $(\% \mathrm{FI})$ was calculated for each challenge, a positive result being $\geq 15 \%$.

A total of 29 subjects had a positive \% fall index, 12 subjects were positive to both challenges, 7 to the room air alone and 10 to the cold air challenge alone. In these subjects $(\mathrm{n}=29)$, the mean (SD) \% fall index to the cold air challenge was 22.7 (11.0)\% compared with 16.3 (9.5)\% for the room air challenge.

We conclude that when employed as a screening test for abnormal airway narrowing, the addition of cold, dry, inspired air during exercise does not increase the number of subjects with a positive response, but the magnitude of the response is increased.

Eur Respir J., 1995, 8, 1314-1317.
*Army Chest Unit, Cambridge Military Hospital, Aldershot, Hants, UK. **AS (Med stats), Ministry of Defence, Stanmore, Middlesex, UK.

Correspondence: D.G. Sinclair Army Chest Unit

Cambridge Military Hospital

Aldershot

Hants GU11 2AN

UK

Keywords: Bronchial challenge cold air

exercise induced asthma

Received: March 31994

Accepted after revision April 91995
In Britain, active asthma is the cause of approximately 50 medical discharges from the army per year, representing one in 3,400 of the serving population. In addition, asthma accounts for approximately 100 hospital admissions and 1,000 noneffective days per year [1]. This study was designed and conducted as part of an attempt to reduce these figures.

Asthma is recognized as a common condition affecting $5-10 \%$ of individuals [2]. Although it is not an impediment to many occupations, army life is unsuitable for asthmatics. Exercise, adverse weather conditions, stress and cigarette smoke are all unavoidable, and soldiers must be fully fit without relying on regular medication. It is, therefore, important to detect active asthmatics at the first screening medical examination that all potential recruits undergo. Our normal practice is to reject as unfit for service, those with active asthma or episodes of wheeze requiring treatment within the previous 4 years.

The situation is further complicated by the fact that asthma occurring during childhood often remits in adolescence, only to return during early adult life, a time when a soldier's career is starting to develop. Therefore, it is important to identify those recruits with a history of asthma who are liable to relapse, whilst not denying entry to those who are able to meet the challenges of a soldier's profession.

History taking and clinical examination are an inadequate screening procedure under these circumstances [1].
It has been standard practice for those with a history of asthma in childhood to undergo an exercise challenge at their maximum heart rate (MHR). They are not accepted for service if the challenge provokes abnormal airway narrowing (see below).

The asthmatic response to exercise is augmented by breathing cold, dry air [3, 4]. The aim of this study was to determine whether breathing cold, dry, inspired air during MHR exercise increased the number of subjects demonstrating abnormal airway narrowing when applied to symptom-free recruits with a history of asthma in childhood.

Subjects and methods

\section{Subjects}

This study is based on a sample of sequentially referred, potential recruits with a history suggestive of asthma in childhood. We were unable to access the medical record of every potential recruit, and relied on a questionnaire completed at the initial medical examination to uncover any history suggestive of this condition, e.g. wheeze, cough, dyspnoea on exertion, and previous use of inhalers. Prior to entry into the study, each subject was interviewed by one of the research team (DGS, NAH, CRW) to confirm, as far as possible, the accuracy of the history. 
Table 1. - Demographic details of patients studied

\begin{tabular}{lc}
\hline Pts n & 100 \\
Sex M/F & $93 / 7$ \\
Age yrs* & $19(15-33)$ \\
Smokers \% & 31 \\
Interval between tests days** & $1(1-18)$ \\
\hline
\end{tabular}

*: value presented as mean, and range in parenthesis; **: value presented as median, and range in parenthesis. Pts: patients; M: male; F: female.

One hundred subjects gave informed consent to participate in the study, which was approved by the Ethics Committee of the Royal Army Medical College. All subjects had been symptom-free and off all treatment for at least four years. Demographic details of all subjects are shown in table 1.

\section{Study design}

This was a prospective study comparing the asthmatic response to MHR exercise whilst breathing room air and cold, dry air in randomized order.

\section{Methods}

Subjects underwent our normal exercise challenge protocol: exercise on an electric treadmill (Case 15, Marquette Electronics Inc, Milwaukee, USA), varying the speed and slope to achieve MHR within 4-6 min of starting, and to maintain it for a further $2 \mathrm{~min}$. The predicted MHR was simply derived by subtracting the subjects age from 220 for males and 200 for females. The protocol of BRUCE [5] was used to guide the timing and increments of change in the speed and slope of the treadmill. All subjects underwent two challenges: breathing room air for one challenge and cold air $\left(-5\right.$ to $\left.-10^{\circ} \mathrm{C}\right)$ for the other, in randomized order. A noseclip was worn by all subjects for both tests, which were, wherever possible, performed on successive days and never on the same day.

Cold air was supplied from a Vacumetrics Turbo Air Challenger (Vacumetrics inc., Vacumed division, Ventura, CA, USA) using compressed air from a size $G$ cylinder. The generation of cold air results from the flow characteristics of the Turbo Air Challenger and the expansion of compressed air. It requires no chemicals or liquids and has been used previously [6]. Cold air was supplied through a standard mouthpiece. Using the fast response digital temperature meter supplied, the inspired air was monitored continuously $12 \mathrm{~cm}$ from the patient's mouth. The water content of the compressed air was $5 \mathrm{mg} \cdot \mathrm{L}^{-1}$ (personal communication, British Oxygen Company). Cooling this to subzero temperatures reduces this to less than $1 \mathrm{mg} \cdot \mathrm{L}^{-1}$. The average water content of the room air has been estimated as $8.5 \mathrm{mg} \cdot \mathrm{L}^{-1}$ (personal communication, London Meteorological Office).

All tests were performed in the morning, at the same time, in the clinical measurement department, supervised by an experienced technician.
The forced expiratory volume in one second (FEV1) of each subject was recorded for each exercise challenge: at rest prior to starting, immediately on completion, and then at 1, 3, 5, 7, 9 and 12 min after completion of each challenge. Measurements were taken to the nearest 0.1 L with a dry wedge spirometer (Vitalograph Medical Instruments $\mathrm{GmbH}$, Hamburg, Germany) using the body temperature, atmospheric pressure and saturation with water vapour (BTPS) scale. A percentage fall index $(\% \mathrm{FI})$ was calculated for each challenge using the formula:

$$
\frac{\text { Resting FEV1 }- \text { Lowest FEV1 }}{\text { Resting FEV1 }} \times 100=\% \text { FI }
$$

A positive result being a $\% \mathrm{FI} \geq 15$ [7].

\section{Statistical methods}

Results within subjects for the room air and cold air challenges were compared using the paired t-test. Differences between the mean \%FI were calculated with 95\% confidence intervals (CI). McNemar's test (incorporating a continuity correction) was used to compare the number of positive results. In addition, the asthmatic responses were analysed using multifactor analysis of variance, estimating the effect of test (i.e. room air versus cold air) within subjects and allowing for any effects attributable to test-sequence or period.

Analysis was conducted using Statgraphics Plus, Version 5.2 [8]. Results are expressed as the mean \pm sD; $p$-values of less than or equal to 0.05 were considered statistically significant.

\section{Results}

All 100 subjects completed both challenges and all FEV1 recordings satisfactorily; each exercising for a total time of $8 \mathrm{~min}$. No subjects were excluded from the study. For administrative reasons, the time between tests exceeded one day in seven subjects. Fifty eight subjects underwent the room air challenge first. There were no significant differences between the two test sequence groups in the distribution of age, cigarette smoking or sex. There was no significant difference between the resting FEV1 obtained immediately before each test, or in the MHR achieved during each test, in any subject. The results are shown in table 2. A total of 29 subjects had a positive \%FI; 12 having a positive response to both challenges, seven being positive to the room air challenge alone, and 10 being positive to the cold air challenge alone. In the subjects with a positive response $(\mathrm{n}=29)$, the mean (SD) \%FI to the cold air challenge was $22.7(11.0) \%$, range $0-44 \%$, compared with $16.3(9.5) \%$, range $0-50 \%$ for the room air challenge, giving a mean difference (cold air - room air) of $6.48 ; 95 \%$ CI 2.1-10.6 $(\mathrm{p}<0.01)$. 
Table 2. - Test details of all subjects $(n=100)$

\begin{tabular}{|c|c|c|c|c|}
\hline & & $\begin{array}{l}\text { Room air } \\
\text { challenge }\end{array}$ & $\begin{array}{c}\text { Cold } \\
\text { challenge }\end{array}$ & $\mathrm{p}$-value \\
\hline Room temperature & ${ }^{\circ} \mathrm{C}$ & $23.3 \pm 3.0$ & $23.5 \pm 3.0$ & NS* \\
\hline "Cold" temperature & ${ }^{\circ} \mathrm{C}$ & - & $-8.3 \pm 2.5$ & \\
\hline Pre-exercise FEV1 & $\%$ pred & $\begin{array}{c}98 \pm 11 \\
(80-110)\end{array}$ & $\begin{array}{c}99 \pm 4 \\
(82-110)\end{array}$ & NS* \\
\hline MHR achieved & $\%$ & $\begin{array}{c}99 \pm 3 \\
(92-105)\end{array}$ & $\begin{array}{c}99 \pm 4 \\
(91-108)\end{array}$ & NS* \\
\hline $\begin{array}{l}\text { Subjects in which } \\
\% \mathrm{FI} \geq 15^{\dagger}\end{array}$ & $\mathrm{n}$ & 19 & 22 & NS** \\
\hline
\end{tabular}

Data are presented as mean $\pm \mathrm{SD}$ and range in parenthesis where appropiate. *: paired t-test; **: McNemar's test with continuity correction. NS: not statistically significant; FEV1: forced expiratory volume in one second; MHR: maximum predicted heart rate: $\%$ : fall index $\geq 15, \mathrm{n}=29$.

\section{Discussion}

This study demonstrates that an abnormal airway response to exercise persists in a number of symptomfree recruits with a history of asthma in childhood. The addition of cold, dry inspired air to exercise did not increase the proportion of this study population developing abnormal airway narrowing. However, in those subjects demonstrating an abnormal response to exercise, the mean \%FI was significantly greater when breathing cold air. This latter finding is in agreement with previous studies [3,9]. The difference between mean \%FI when breathing cold or room air was smaller than that reported by STRAuss et al. [3]. This may reflect the differing populations studied; our population was free of symptoms, whereas Strauss et al. [3] studied patients known to have active exercise-induced asthma (EIA).

There was a considerable degree of overlap between the two tests; the number of positive responders for the two challenges was greater than for either alone. We hypothesized that some subjects negative to the room air challenge would be positive to the cold air challenge, and indeed this was the case. We did not, however, expect some subjects to be positive to the room air challenge and negative to the cold air challenge. It has been demonstrated that with random exercise testing of symptomatic asthmatics, of average severity, only $70-80 \%$ will develop a postexercise change in lung function which is outside the normal range [10]. This observation and the fact that our subjects were asymptomatic, with mild disease at worst, may account for the inconsistent response to either of the two challenges.

Exercise was used to assess bronchial responsiveness as it is safe, quick, simple and similar to the physical nature of army life. There is also evidence to suggest that as asthmatic children grow out of their clinically active disease their responsiveness to exercise decreases, whilst their responsiveness to inhaled pharmacological agents persists $[11,12]$ In these circumstances, an inhaled challenge in a recruit with a history of asthma in childhood would, we feel, be inappropriate as it would not discriminate between a history of asthma and active disease.
The precise trigger for EIA remains uncertain, but is thought to be related to either drying of the airways with a subsequent increase in the osmolarity of the lining fluid $[13,14]$, or reactive hyperaemia of the tracheobronchial capillary bed induced by rapid rewarming of the airways after exercise-induced cooling [15]. Whatever the mechanism, the severity of EIA is known to be influenced by the level of work undertaken, together with the temperature and water content of the inspired air [16-18]. It was, therefore, logical to assess whether the use of cold, dry, inspired air would increase the sensitivity of the exercise challenge. In previous studies, exercise to $90 \%$ of MHR has been recommended [19, 20]. We found it simpler to use an incrementally increasing work rate aimed at achieving the age-derived MHR of the subject. This provided a clear end-point, did not cause any morbidity in our population, and ensured that every subject achieved at least $90 \%$ of their predicted MHR.

The standardization of the exercise protocol to pulse rate rather than ventilation may explain some of the differences between the two tests. Had we measured the rate of ventilation and ensured that $50 \%$ of predicted maximum voluntary ventilation $(\mathrm{FEV} 1 \times 35)$ was sustained for $4 \mathrm{~min}$ of exercise, we could be more confident that the enhancement of the airway response was, in fact, due to the conditions of the inspired air. Under these circumstances, it is possible that the discordance between the two tests would have been less.

We appreciate that our methods of subject selection are flawed and that potential recruits may not declare their relevant history. This remains an insurmountable problem without access to previous medical records.

In conclusion, an abnormal degree of airway narrowing following exercise is demonstrable in symptom-free recruits with a history of childhood asthma. The inconsistency of the airway response to exercise suggests that this type of screening is an imperfect tool in this and similar populations.

\section{References}

1. Dickinson JG. Asthma in the army: a retrospective study and review of the natural history of asthma and its implications for recruitment. JR Army Med Corps 1988; 134: 65-73.

2. Fleming DM, Crombie DL. Prevalence of asthma and hayfever in England and Wales. Br Med J 1987; 294: 279-283.

3. Strauss RH, McFadden ER, Ingram RH, Jaeger JJ. Enhancement of exercise-induced asthma by cold air. $N$ Engl J Med 1977; 297: 743-747.

4. Strauss RH, McFadden ER, Ingram RH, Chandler Deal $\mathrm{E}$, Jaegar JJ. Influence of heat and humidity on the airway obstruction induced by exercise in asthma. J Clin Invest 1978; 61: 433-440.

5. Bruce RA. Exercise testing for evaluation of ventricular function. N Engl J Med 1977; 296: 671-675.

6. Reisman J, Mappa L, de Benedictis F, McLaughlin J, Levison H. Cold air challenge in children with asthma. Paed Pulmonol 1987; 3: 251-254.

7. Anderson SD. Diagnosis and management of exerciseinduced asthma. In: Gershwin ME, Halpern GM, eds. 
Bronchial asthma: Principles of Diagnosis and Treatment. New Jersey, Humana Press, 1994; pp. 513-547.

8. Statistical Graphics Corporation. Statgraphics Plus, version 5.2. STSC, inc., 1991.

9. Deal EC, McFadden JR, Ingram RH, Strauss RH, Faeger JJ. Role of respiratory heat exchange in production of exercise induced asthma. J Appl Physiol: Respirat Environ Exercise Physiol 1979, 46: 467-475.

10. Godfrey S, Springer C, Noviski N, Maayan Ch, Avital A. Exercise but not Methacholine differentiates asthma from chronic lung disease in children. Thorax 1991; 46: 488-492.

11. Balflour-Lynn L, Tooley M, Godfrey S. A study comparing the relationship of exercise-induced asthma to clinical asthma in childhood. Arch Dis Child 1980; 56: 450-454.

12. Martin AJ, Landau LI, Phelan PD. Lung function in young adults who had asthma in childhood. Am Rev Respir Dis 1980; 122: 609-616.

13. Hahn A, Anderson SD, Morton AR, Black JL, Fitch KD A reinterpretation of the effect of temperature and water content of the inspired air in exercise-induced asthma. Am Rev Respir Dis 1984; 130: 575-579.
14. Rimmer J, Bryant DH. Effect of hypo- and hyperosmolarity on basophil histamine release. Clin Allergy 1986; 16: 221-230.

15. McFadden ER. Hypothesis: exercise-induced asthma as a vascular phenomenon. Lancet 1990; 335: 880883.

16. Weinstein RE, Anderson JA, Kvale P, Sweet LC. Effects of humidification on exercise induced asthma (EIA). J Allergy Clin Immunol 1976; 57: 250-251.

17. Bar-Or O, Neuman I, Dotan R. Effects of dry and humid climates on exercise-induced asthma in children and adolescents. J Allergy Clin Immunol 1977; 60: 163168.

18. Chen WY, Horton DJ. Heat and water loss from the airways and exercise induced asthma. Respiration 1977; 34: 305-313.

19. Cropp GJA. The exercise bronchoprovacation test: standardisation of procedures and evaluation of response. $J$ Allergy Clin Immunol 1979; 64: 627-633.

20. Eggleston PA, Rosenthal RR, et al. Guidelines for the methodology of exercise challenge testing of asthmatics. J Allergy Clin Immunol 1979; 64: 642-645. 